Dermatology

Edited by Vanessa Venning DM FRCP Consultant Dermatologist,

Churchill Hospital, Oxford, and Peter Mortimer MD FRCP Professor of

Dermatological Medicine, St George's Hospital Medical School, London

\section{Connective tissue}

\section{disease and the skin}

Sabine Sommer MD MRCP, Specialist

Registrar in Dermatology

Mark JD Goodfield MD FRCP, Consultant Dermatologist

Department of Dermatology, Leeds General Hospital

\section{Clin Med JRCPL 2002;2:9-14}

Connective tissue disorders are uncommon conditions of unknown aetiology. They occur more often in women, affect skin and joints and at times also other organs, and are associated with the production of autoantibodies. They have a genetic background, but are probably induced by a variety of environmental stimuli (Table 1$)^{1}$. This review focuses on cutaneous manifestations.

\section{Lupus erythematosus}

Cutaneous lupus erythematosus (LE) occurs in discoid (DLE), systemic (SLE) and intermediate subacute cutaneous
(SACLE) variants. These types are clinically similar and histologically indistinguishable, with similar laboratory abnormalities (albeit to a lesser extent in DLE). However, there is a different age and sex distribution and the genetic associations are different. The risk of transformation of DLE to SLE overall is only $5 \%$, but HLA B8-positive middleaged women are at greater risk ${ }^{2}$.

\section{Discoid lupus erythematosus}

DLE affects twice as many women as men, mostly in their 30s, and takes a chronic relapsing course. In genetically predisposed individuals, the disease may be precipitated by a variety of environmental factors including trauma, stress, infections and particularly ultraviolet light ${ }^{1}$.

Table 1. Triggers of connective tissue diseases.

- Trauma

- Sunburn

- Exposure to cold

- Hormonal changes

- Mental stress

- Infection

- Drugs

- Chemicals

\section{Key Points}

Connective tissue diseases are triggered by environmental stimuli in genetically predisposed patients

Women are more commonly affected than men

Skin manifestations are often the presenting feature of a multisystem disorder

Diagnosis and classification are not always easy; incomplete, as well as overlap, syndromes exist

Treatments are not curative, but disease control may be achieved by topical or systemic immune modulatory drugs

KEY WORDS: CPD, lupus, scleroderma, dermatomyositis, cutaneous disease
DLE most commonly involves the face and scalp, but disseminated forms occur (Fig 1), and mucosa and nails may be involved. Red scaly patches heal with scarring and pigmentary change. Removal of the scales reveals horny plugs filling the follicular openings. Scarring alopecia is a problem for a third of patients. Raynaud's phenomenon and joint pains may occur. Subcutaneous involvement (lupus profundus) and lesions resembling erythema multiforme are unusual presentations.

The diagnosis of DLE depends on the characteristic clinical findings of photoexacerbated scaly lesions with follicular plugging and scarring, combined with typical histology of a lymphocytic infiltrate around dermal vessels and appendages and vacuolation of the basal layer of the epidermis.

\section{Treatment}

First-line treatment consists of a sunblock and a potent topical steroid, for example, clobetasol propionate or intralesional steroid injections. Failing this, oral antimalarials alone (usually hydroxychloroquine) or in combination with topical steroids should be tried. The list of third-line treatments is long and poorly evaluated, but includes thalidomide, oral gold, oral retinoids, dapsone and immunosuppressants for severe cases ${ }^{3}$.

\section{Systemic lupus erythematosus}

SLE is a chronic episodic disease, affecting all races but is particularly common in young black American women. It has a peak onset in early adult life and a female to male ratio of 8:1.

Diagnostic criteria are summarised in Table 2. There is impaired cell-mediated immunity and an exaggerated humoral response with the production of antinuclear antibodies. The role of autoantibodies in pathogenesis remains unclear. Some antibodies are more disease-specific (Table 3). Anti-Ro antibodies are implicated in the development of the characteristic rash seen in subacute cutaneous LE and neonatal LE. They are also responsible for producing the 


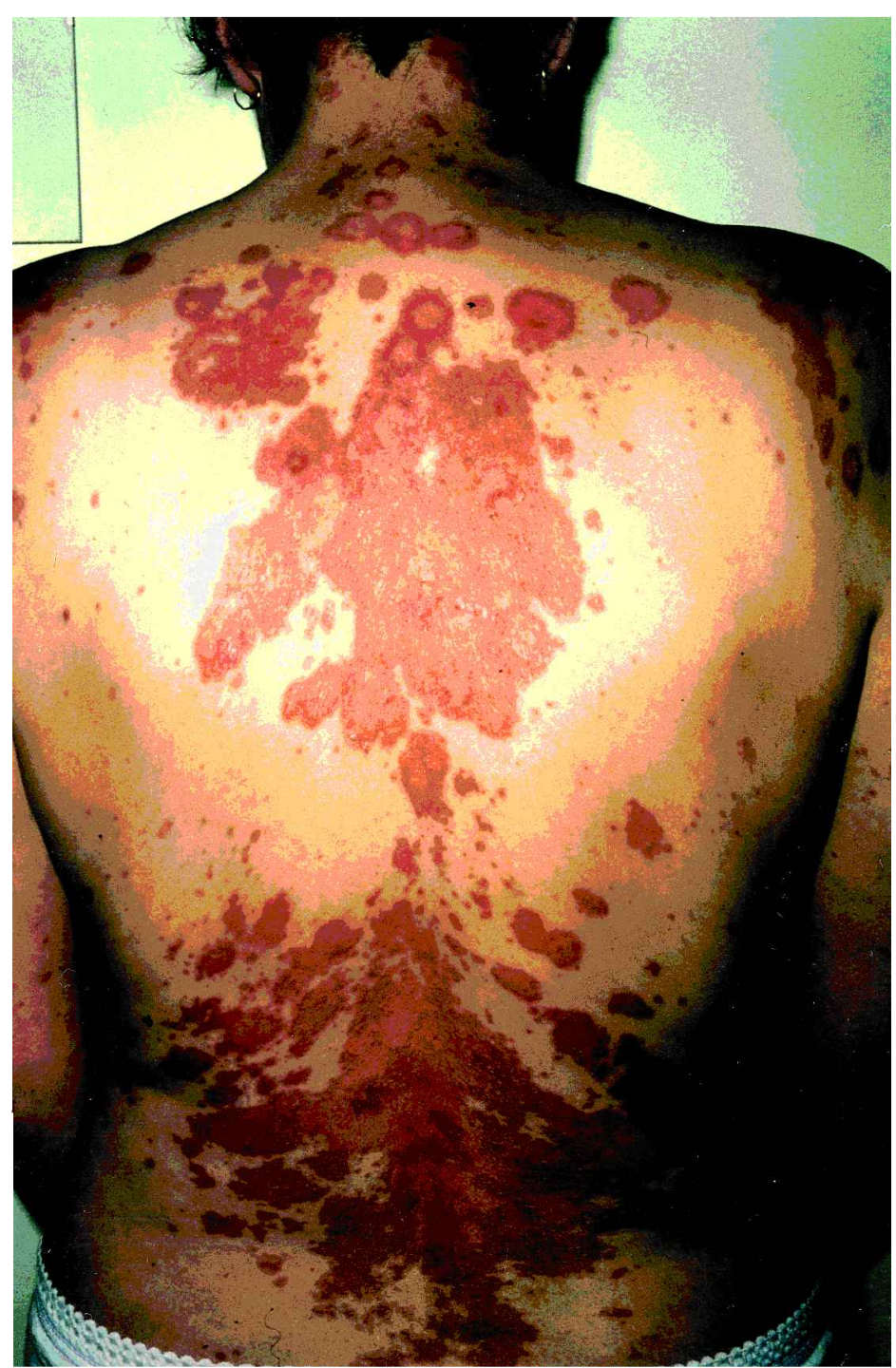

Fig 1. Disseminated erythematosus squamous plaques of discoid lupus erythematosus.

Table 2. American Rheumatism Association criteria for diagnosis of systemic lupus erythematosus (four of these features are necessary to diagnose SLE).

1 Malar rash

2 Discoid rash

3 Photosensitivity

4 Oral ulcers

5 Non-erosive arthritis

6 Serositis: pleurisy or pericarditis

7 Renal disorder: persistent proteinuria (>0.5 g/day) or cellular casts

8 Neurological disorder: seizures or psychosis

9 Haematological disorder: haemolytic anaemia, leukopenia $(>4,000 / \mathrm{mm})$, lymphopenia $(<1,500 / \mathrm{mm})$ or thrombocytopenia $(>100,000 / \mathrm{mm})$

10 Immunological disorder: LE cells, false +ve syphilis serology ( $>6$ month), anti-DNA or anti-Sm antibody

11 Antinuclear antibodies congenital heart-block seen in this condition ${ }^{4}$. In addition to the same exacerbating factors as seen in DLE, there is also a drug-induced form.

Skin involvement occurs in $80 \%$ of patients with SLE and is the presenting sign of disease in up to $25 \%$ of patients ${ }^{5}$. The commonest skin sign is a photosensitive facial erythema with fine scaling (the so-called butterfly rash) (Fig 2 ), but the presentation may be diverse (Table 4$)^{6}$.

\section{Treatment}

Photoprotection and medications used to treat DLE may also be useful in SLE, although antimalarials are generally less effective. The management of the skin in SLE is usually dictated by the need to control the systemic manifestations of the disease.

\section{Intermediate subacute cutaneous variants $^{7}$}

There are two well recognised patterns of skin disease in this subtype. The first, and most common, has red annular lesions with central clearing. Lesions are usually made worse by light and 75\% of patients are Ro-antibody positive. The second variant is indistinguishable from widespread DLE, Ro-antibodies tend to be positive, patients may have associated arthritis but rarely develop severe internal organ disease.

\section{Treatment}

Sun protection is critical in the management and lesions respond rapidly to oral steroids or dapsone ${ }^{7}$.

\section{The antiphospholipid antibody syndrome $^{8}$}

About 23\% of patients with SLE have antiphospholipid antibodies, some of which interfere with components of the coagulation cascade (the so-called 'lupus anticoagulant'), although similar antibodies occur in patients without SLE. A proportion of patients with the lupus anticoagulant develop the antiphospholipid syndrome with paradoxical

LE = lupus erythematosus. 
Table 3. Percentage of patients with non-organ-specific autoantibodies in connective tissue diseases.

\begin{tabular}{|c|c|c|c|c|c|c|}
\hline & ANA/ANF & Nuclear RNP & $\begin{array}{l}\text { Ro (SSA) } \\
\text { La (SSB) }\end{array}$ & $\mathrm{Sm}$ (ENA) & $\begin{array}{l}\text { tromere } \\
\text { (\%) }\end{array}$ & Other \\
\hline DLE & $\begin{array}{l}35 \% \text { (homogeneous, } \\
\text { speckled) }\end{array}$ & & Rare & & & \\
\hline SACLE & $\begin{array}{l}80 \% \text { (homogeneous, } \\
\text { speckled) }\end{array}$ & & $75 \%$ & & & \\
\hline SLE & $\begin{array}{l}100 \% \text { (homogeneous, } \\
\text { speckled) }\end{array}$ & & Sometimes & $30 \%$ & 6 & $\begin{array}{l}\text { Drug-induced cases: } \\
\text { histones } 50-70 \% \\
\text { double-stranded DNA I }\end{array}$ \\
\hline Systemic sclerosis & $90 \%$ (speckled, nucleolar) & & & & 50 & $\begin{array}{l}20 \% \text { Topoisomerase } \\
\text { (SCl 70) }\end{array}$ \\
\hline $\begin{array}{l}\text { Mixed connective } \\
\text { tissue disease }\end{array}$ & $100 \%$ (speckled) & High titre diagnostic & & $100 \%$ high titre & 6 & \\
\hline Dermatomyositis & Sometimes (speckled) & Sometimes & & & & $20 \%$ Jo 1 \\
\hline
\end{tabular}

ANA = antinuclear antibody; ANF = antinuclear factor; DLE = discoid lupus erythematosus; ENA = extractable nuclear antigens;

RNP = ribonucleoprotein; SACLE = subacute cutaneous lupus erythematosus; SLE = systemic lupus erythematosus.

thrombotic episodes. In the skin, these may present as livedo reticularis, thrombophlebitis, ulcers and skin infarcts or gangrene.

\section{Scleroderma}

Sclerosis of the skin (scleroderma) is a symptom, not a diagnosis. Cutaneous sclerosis is a characteristic feature of progressive systemic sclerosis but also occurs in several other settings (Table 5).

Table 4. Cutaneous features of systemic lupus erythematosus in 73 patients $^{6}$.

\begin{tabular}{ll} 
Cutaneous feature & $\%$ \\
\hline Photosensitivity & 63 \\
Raynaud's phenomenon & 60 \\
Butterfly rash & 51 \\
Urticarial lesion (>36 hours) & 44 \\
Non-scarring alopecia & 40 \\
Mouth ulceration & 31 \\
Chronic DLE & 25 \\
Chilblain lupus & 20 \\
Scarring DLE alopecia & 14 \\
Bullous eruptions & 8 \\
SACLE & 7 \\
Facial oedema & 4 \\
Livedo reticularis & 4 \\
Episcleritis & 4 \\
Cheilitis & 4 \\
Cutaneous vasculitis & 1 \\
\hline
\end{tabular}

DLE = discoid lupus erythematosus; SACLE = subacute cutaneous lupus erythematosus.

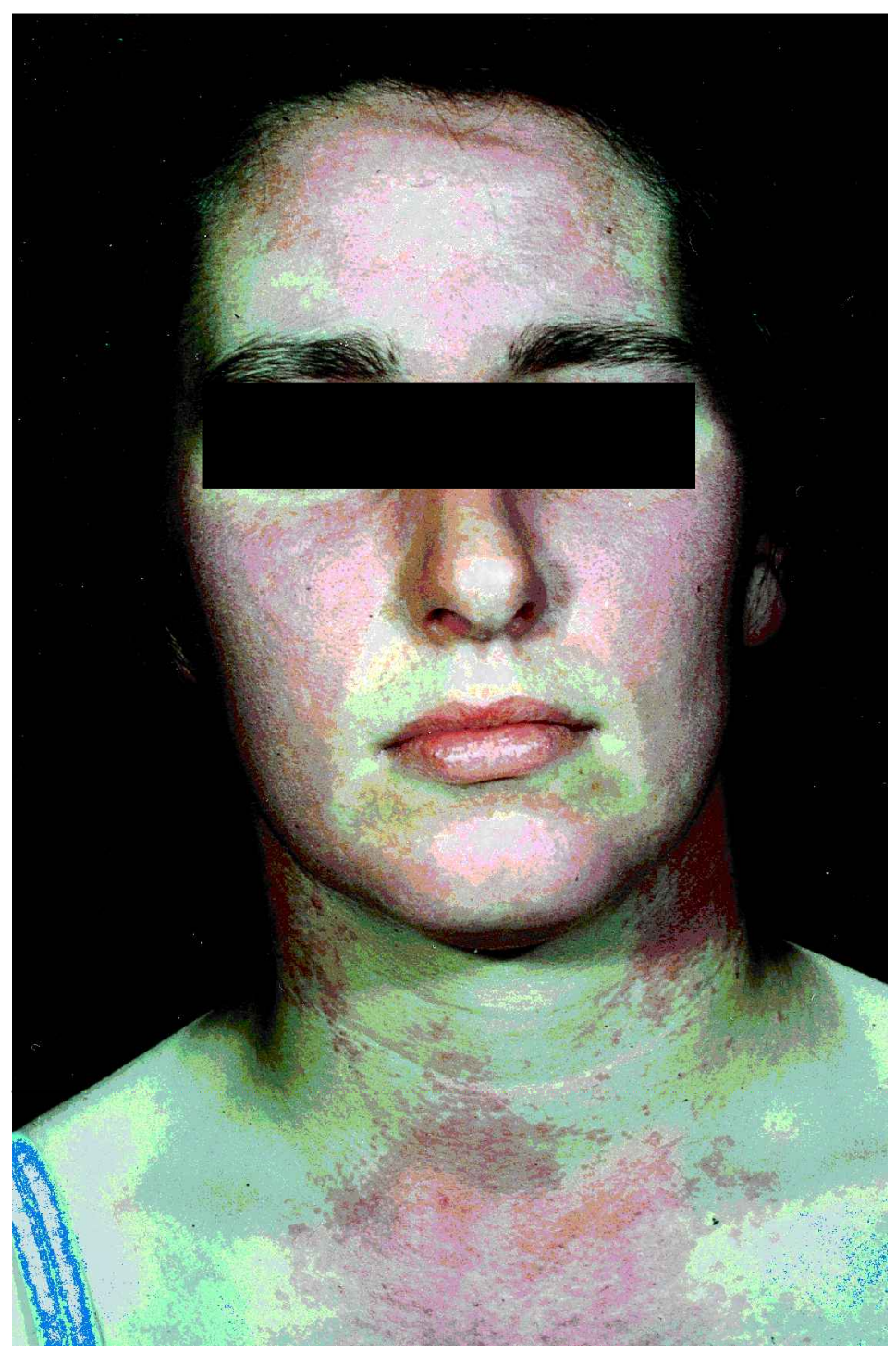

Fig 2. Butterfly rash of systemic lupus erythematosus. 
Scleroderma produces indurated, hard, shiny, tethered skin with loss of appendages (ie hair and sweat glands).

\section{Morphoea}

Morphoea, or localised scleroderma, usually presents as a plaque of indurated mauve skin which later becomes waxy and either pale or hyperpigmented with absent hair and sweating (Fig 3). Young adults aged 20-40 are affected, with a threefold preponderance in women. The cause is unknown but may be provoked by trauma or infection.

Linear morphoea may involve subcutaneous tissues producing atrophy, as in en coup de sabre morphoea. Extensive morphoea may mimic system sclerosis, but differs in the absence of systemic disease, Raynaud's phenomenon and hand lesions. Autoantibodies are usually negative. Plaques around joints can cause contractures and impaired mobility.

\section{Treatment}

Plaque morphoea lesions may improve spontaneously, but local steroids, topical and oral vitamin $\mathrm{D}$ analogues, local or systemic psoralen ultraviolet A (PUVA) light therapy can be used.

Table 5. Causes of cutaneous sclerosis.

- Progressive systemic sclerosis

- Morphoea

- Chronic venous hypertension (lipodermatosclerosis)

- Shoulder-hand syndrome (fingers)

- Myeloma, paraproteinaemia

- Primary systemic amyloidosis

- Hypothyroidism

- Eosinophilic fasciitis

- Porphyria cutanea tarda

- Carcinoid syndrome (legs)

- Occupational exposure eg PVC, organic solvents (perchlorethylene used in dry cleaning)

- latrogenic eg chronic GVH disease, bleomycin

$\mathrm{GVH}=$ graft versus host disease

PVC $=$ polyvinyl chloride

\section{Eosinophilic fasciitis ${ }^{9}$}

Eosinophilic fasciitis, a scleroderma-like syndrome, appears to be a separate entity. It presents with pain and swelling of the distal limbs, followed by deep induration, often accompanied by eosinophilia both within the lesion and in the blood. Biopsy shows dermal sclerosis with inflammation and fibrosis of the deep fascia. Spontaneous remissions occur and systemic steroids are helpful.

\section{Systemic sclerosis}

Diagnostic criteria of this rare multisystem disorder are outlined in Table $6^{10}$.
The female to male ratio is between 3 and 6:1, with a peak onset in the 40 s. There is debate over the target tissue but the vascular endothelial cell appears to be involved early in the disease, leading to vascular occlusion, tissue ischaemia and fibrosis due to increased collagen formation. The observed autoimmunity may be a primary abnormality or due to cell damage. Antinuclear antibodies are almost invariably present and extractable nuclear antigens occur. Anti-centromere antibodies occur in patients with 'limited' disease in whom sclerosis tends to be peripheral and internal organ involvement uncommon. SCl 70 (antitopoisomerase) occurs with severe disease.

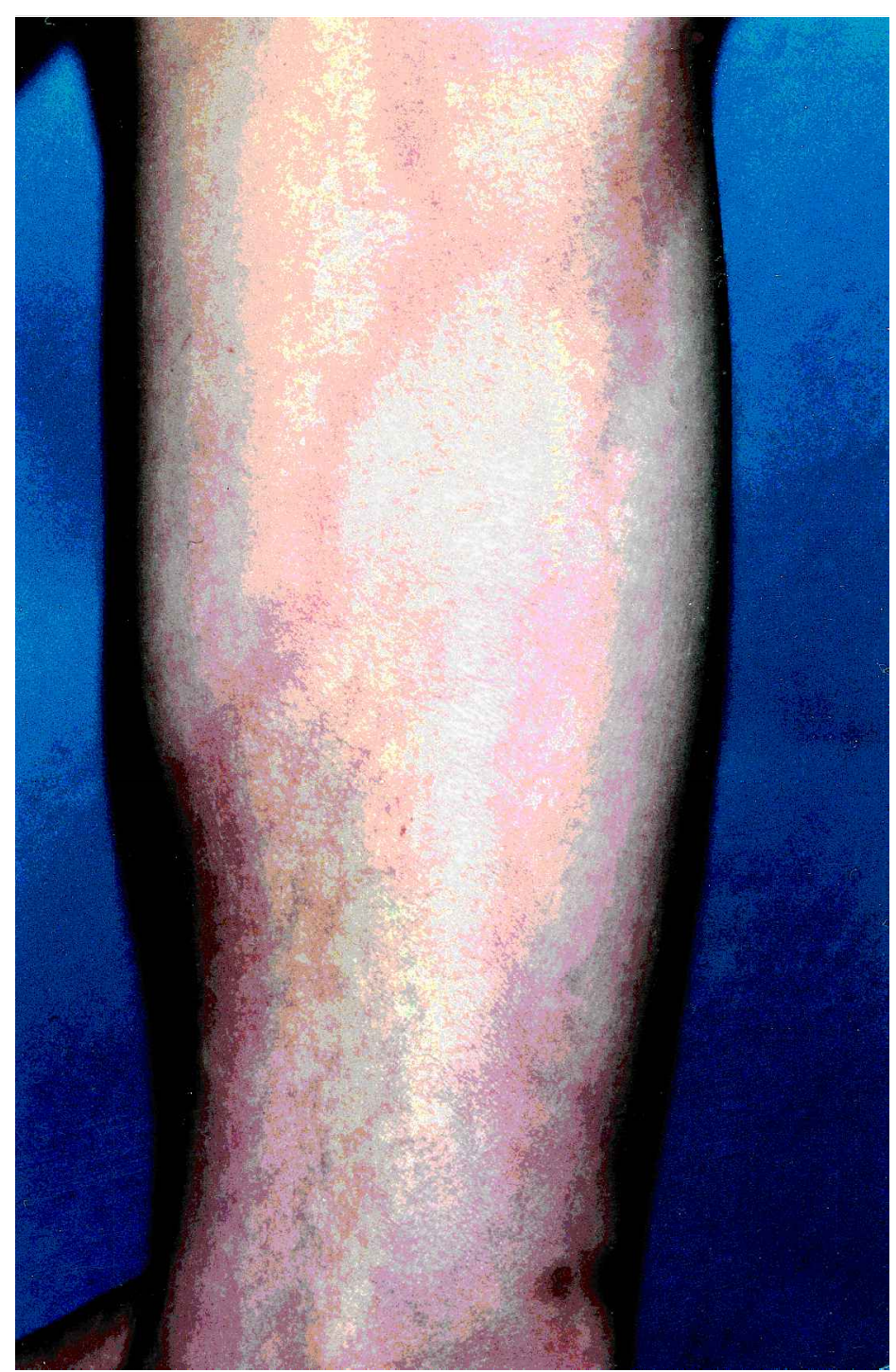

Fig 3. Plaque of morphoea, showing central white atrophy and surrounding mauve border. 
Table 6. American Rheumatism Association criteria for the diagnosis of systemic sclerosis.

\begin{tabular}{ll} 
Criterion & Features \\
\hline Major & $\begin{array}{l}\text { Scleroderma proximal to } \\
\text { the digits, or at least } 2 \text { of } \\
\text { the minor criteria }\end{array}$ \\
Minor & (i) Sclerodactyly \\
& (ii) Digital pitted \\
& (iii) Bilateral basal \\
&
\end{tabular}

Unlike morphoea, the skin involvement is generalised and irreversible. Internal organ involvement is variable. Clinical features include Raynaud's phenomenon, early swelling and later hardening and tightening of the skin of the fingers, frequently with cutaneous calcinosis and digital ulceration (Fig 4). Facial involvement produces loss of expression lines, a beaked nose and restricted opening of the mouth. Telangiectasia, and hyper- or hypopigmentation are seen. Oesophageal involvement causes dysphagia and gastro-oesophageal reflux. The prognosis depends on the degree of internal organ involvement.

\section{Treatment}

Currently, no treatment is known to alter the outcome of the disease, but symptomatic management is important. Oral steroids, penicillamine, colchicine, vasodilators, immunosuppressants, prostacyclin infusions and phototherapy have all been used with symptomatic benefit ${ }^{11}$.

\section{Mixed connective tissue disease $^{12}$}

The presence of the U1-RNP antibody defines mixed connective tissue disease which has features of SLE, systemic sclerosis, dermatomyositis and rheumatoid arthritis. Evolution into a definite disease entity may occur but overall prognosis tends to be good.

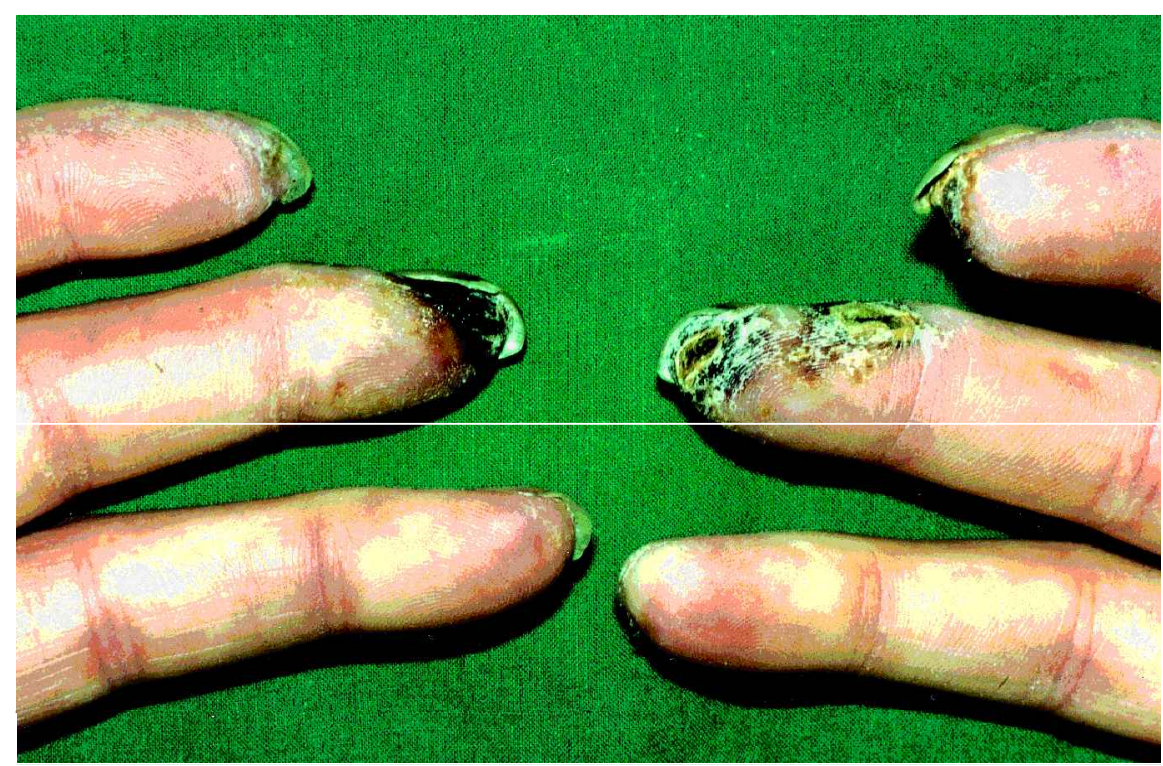

Fig 4. Spindling of the fingers and digital infarcts in systemic sclerosis.

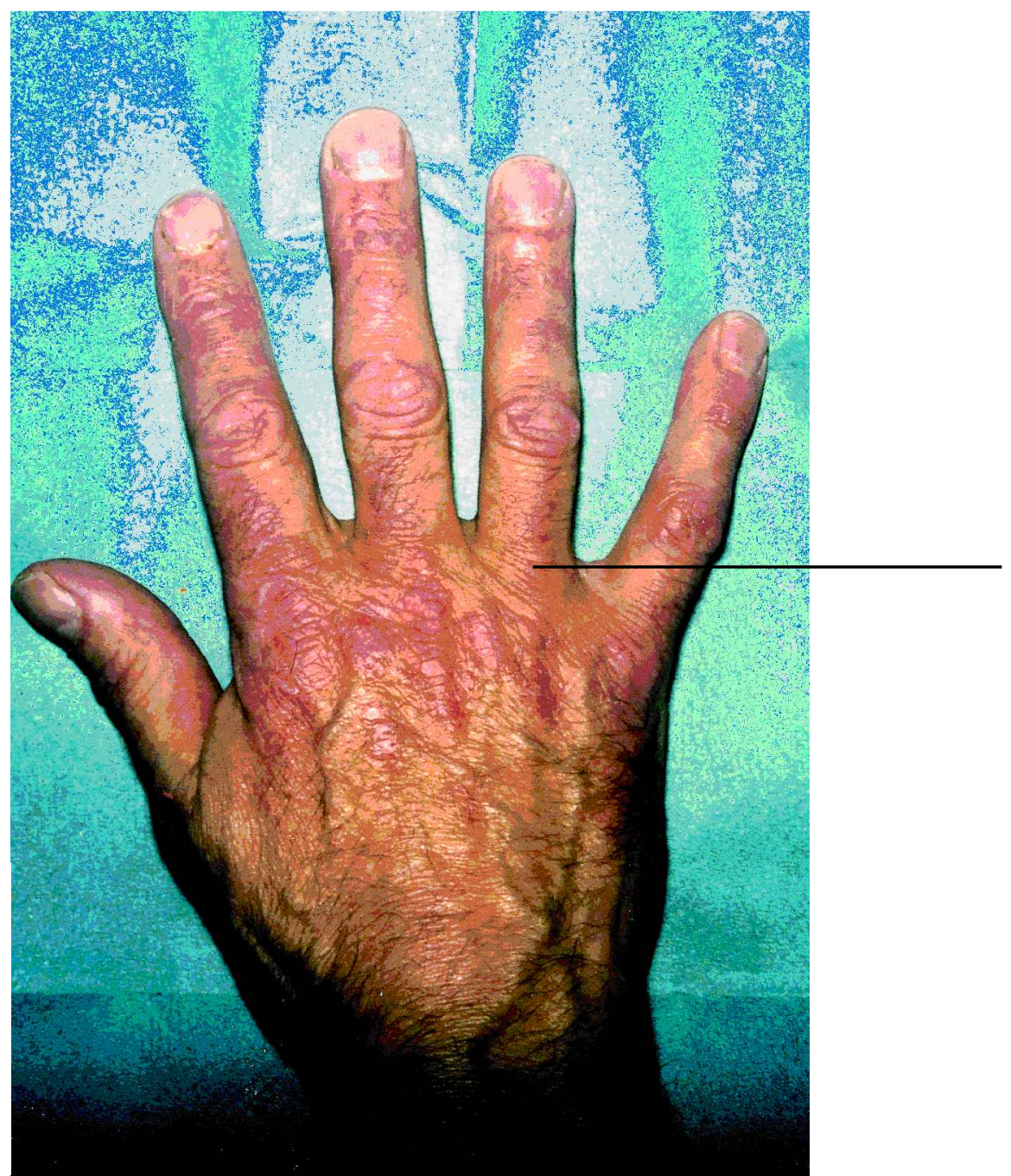

Fig 5. Gottron's papules overlying knuckles in dermatomyositis. 


\section{Dermatomyositis}

Dermatomyositis has a peak onset before the age of 10 and a second peak between 40 and 60 years. Mauve-red ('heliotrope') is the distinctive colour of the rash and oedema is prominent. Photosensitivity frequently produces puffy mauve discolouration of the eyelids and other areas. Flat topped lesions overlie the knuckles and other bony prominences (Gottron's papules) (Fig 5). The nailfolds show dilated capillary loops and ragged cuticles. Less typical are bullous, urticarial or psoriasiform lesions or reticulate telangiectatic erythema with atrophic scarring and pigmentary changes (poikiloderma). Vasculitis and panniculitis can occur, while calcinosis may follow or occur spontaneously. Calcinosis is a good sign for survival, but makes functional recovery less likely. It affects more than $50 \%$ of children and $15 \%$ of adults with dermatomyositis. Any or all muscle types, including respiratory and cardiac, may be involved. A rheumatoid pattern of arthritis and pulmonary fibrosis occurs, particularly in those with anti-Jo1 antibodies.

The diagnosis depends upon the association of a typical rash with muscle weakness, although muscle and skin biopsy can be normal. Electromyography, muscle enzyme levels and magnetic resonance imaging may be helpful in establishing the presence of muscle disease. Early blood vessel damage appears to be secondary to the presence of anti-endothelial cell antibodies. Circulating immune complexes have been demonstrated in 70\% of patients. An associated malignancy is present in $25-40 \%$ of patients over 40 years; it is possible that an abnormal immunological response to the neoplasm may cause the disease. Childhood dermatomyositis is not associated with neoplasia ${ }^{13}$. Two-thirds of deaths are due to underlying malignancy, the rest to pulmonary or cardiac failure.

\section{Treatment}

Treatment of the underlying malignancy is essential. Systemic steroids, azathioprine and methotrexate are helpful and may be required for many years. Not all patients respond to therapy.

\section{References}

1 Rowell NR, Goodfield MJD. The 'connective tissue diseases'. In: Champion $\mathrm{RH}$, Burton JL, Burns DA, Breathnach SM (eds). Textbook of dermatology. 6th edn. Oxford: Blackwell Science, 1998:2437-76.

2 Scott A, Rees EG. The relationship of systemic lupus erythematosus and discoid lupus erythematosus. Arch Dermatol 1959; 79:422-35.

3 Hasper MF. Chronic cutaneous lupus erythematosus. Thalidomide treatment of 11 patients. Arch Dermatol 1983;119:812-5.

4 Petri M, Watson R, Hochberg MC. Anti-Ro antibodies and neonatal lupus. Review.
Rheum Dis Clin North Am 1989;15:335-60.

5 Tuffanelli DL, Dubois EL. Cutaneous manifestations of systemic lupus erythematosus. Arch Dermatol 1964;90:377-86.

6 Yell JA, Mbuagbaw J, Burge SM. Cutaneous manifestations of systemic lupus erythematosus. Br J Dermatol 1996;135:355-62.

7 Callen JP, Klein J. Subacute cutaneous lupus erythematosus. Clinical, serologic, immunogenetic, and therapeutic considerations in seventy-two patients. Arthritis Rheum 1988;31:1007-13.

8 Khamashta MA, Hughes GR. Antiphospholipid syndrome. Br Med J 1993;307: 883-4.

9 Bennett RM, Herron A, Keogh L. Eosinophilic fasciitis. Case report and review of the literature. Ann Rheum Dis 1977;36:354-9.

10 Preliminary criteria for the classification of systemic sclerosis (scleroderma). Subcommittee for scleroderma criteria of the American Rheumatism Association Diagnostic and Therapeutic Criteria Committee. Arthritis Rheum 1980;23: 581-90.

11 Alarcon-Segovia D, Ibanez G, Kershenobich D, Rojkind M. Letter: Treatment of scleroderma. Lancet 1974;i:1054-5.

12 Rowell NR. Overlap in the connective tissue diseases. Semin Dermatol 1985;4:136-42.

13 Maugars YM, Berthelot JM, Abbas AA, Mussini JM, et al. Long term prognosis of 69 patients with dermatomyositis or polymyositis. Clin Exp Rheumatol 1996;14: 263-74.

Address for correspondence: Dr Sabine Sommer, Specialist Registrar, General Infirmary, Great George Street, Leeds LS1 3EX 